\title{
Some Identities Involving the Higher-Order Changhee Numbers and Polynomials
}

\author{
Nanding Wuyungaowa \\ Department of Mathematics, College of Sciences and Technology, Inner Mongolia University, Huhhot, China \\ Email: 1141384395@qq.com
}

How to cite this paper: Wuyungaowa, N. (2018) Some Identities Involving the Higher-Order Changhee Numbers and Polynomials. Journal of Applied Mathematics and Physics, 6, 647-656. https://doi.org/10.4236/jamp.2018.64057

Received: November 24, 2017

Accepted: April 9, 2018

Published: April 12, 2018

\begin{abstract}
In this paper, by the classical method of Riordan arrays, establish several general involving higher-order Changhee numbers and polynomials, which are related to special polynomials and numbers. From those numbers, we derive some interesting and new identities.
\end{abstract}

\section{Keywords}

Riordan Array, Changhee Number, Changhee Polynomial

\section{Introduction}

Recently, many works have been devoted to the study of Changhee number identities by various method [1] [2] [3] [4]. In [1], D.S.Kim and T.Kim give various identities of the higher-order Changhee numbers and polynomials which are derived from umbral calculas. In [3], J.Kwon consider Witt-type formula for the weighted Changhee numbers and polynomials. In [4], D.S.Kim and T.Kim also introduced the non-linear Changhee differential equations and these differential equations turned out to be very useful for special polynomials and mathematical physics and so on. In the present paper, we make use of the Riordan arrays method in a constructive way to establish some general summation formulas, from which series of Changhee numbers and polynomials identities can be obtained. In particular, besides the Changhee numbers, some identities also involve the Stirling numbers of both kinds, Daehee numbers of both kinds, Lah numbers, Harmonic numbers, Genocchi numbers and polynomials and Euler polynomials. It can be found that no Changhee number identities presented in [1] [2] [3] [4] referred to above have other special combinatorial sequences, and actually, there are not many identities involving both Changhee numbers and other combinatorial numbers in the literature. From this point of view, our re- 
sults extend the range of Changhee number and polynomials identities.

The study of this paper follows D.S.Kim and T.Kim, s result [1]. Let $\mathrm{p}$ be an odd prime number. Throughout this paper, $\mathbb{Z}_{p}, \mathbb{Q}_{p}, \mathbb{C}_{p}$ will denote the ring of $\mathrm{p}$-adic integers, the field of $\mathrm{p}$-adic numbers and the completion of the algebraic closure of $\mathbb{Q}_{p}$. The p-adic norm ||$_{p}$ is normalized as $|p|_{p}$. Let $f \in C\left(\mathbb{Z}_{p}\right)$ be the space of continuous functions on $\mathbb{Z}_{p}$.

For $f \in C\left(\mathbb{Z}_{P}\right)$, the fermionic $\mathrm{p}$-adic integral on $\mathbb{Z}_{P}$ is defined by Kim to be

$$
I_{-1}=\int_{\mathbb{Z}_{p}} f(x) d \mu_{-1}(x)=\lim _{N \rightarrow 0} \sum_{x=0}^{p^{N}-1} f(x)(-1)^{x} .
$$

For $f_{1}(x)=f(x+1)$, we have

$$
I_{-1}\left(f_{1}\right)+I_{-1}(f)=2 f(0) .
$$

For $t \in \mathbb{C}_{p}$ with $\left|t_{p}\right|<p^{-\frac{1}{p-1}}$, the Changhee polynomials of the first kind are given by the fermionic $\mathrm{p}$-adic integral on $\mathbb{Z}_{p}$ :

$$
\int_{\mathbb{Z}_{p}}(1+t)^{x+y} d \mu_{-1}(y)=\frac{2}{(1+t)+1}(1+t)^{x}=\sum_{n=0}^{\infty} C h_{n}(x) \frac{t^{n}}{n !},
$$

In special case, when $x=0, C h_{n}=C h_{n}(0)$ are called the Changhee numbers of the first kind.

From Equation (1), we note that

$$
\int_{\mathbb{Z}_{p}}(x+y)_{n} d \mu_{-1}(y)=C h_{n}(x),
$$

where $(x)_{n}=x(x-1) \cdots(x-n+1),(n \geq 1),(x)_{0}=1$.

For $r \in \mathbb{N}$, Changhee polynomials of the first kind with order $r$ by defined by the generating function as follows:

$$
\begin{aligned}
& \int_{\mathbb{Z}_{p}} \cdots \int_{\mathbb{Z}_{p}}(1+t)^{x_{1}+\cdots x_{r}+x} d \mu_{-1}\left(x_{1}\right) \cdots d \mu_{-1}\left(x_{r}\right) \\
& =\left(\frac{2}{2+t}\right)^{r}(1+t)^{x}=\sum_{n=0}^{\infty} C h_{n}^{(r)}(x) \frac{t^{n}}{n !}
\end{aligned}
$$

where $n$ is a nonnegative integer. In special case, when $x=0, C h_{n}^{(r)}=C h_{n}^{(r)}(0)$ are called the Changhee numbers of the first kind with order $r$.

It is not difficult to show that

$$
\left(\frac{2}{2+t}\right)^{r}=\sum_{n=0}^{\infty}\left(-\frac{1}{2}\right)^{n}\left(\begin{array}{c}
r+n-1 \\
n
\end{array}\right) t^{n} .
$$

From Equation (5) and Equation (6), we have

$$
C h_{n}^{(r)}=\frac{n !}{2^{n}}\left(\begin{array}{c}
n+r-1 \\
r-1
\end{array}\right)
$$

For $t \in \mathbb{C}_{p}$ and $|t|_{p}<p^{-\frac{1}{p-1}}$, Changhee polynomials of the second kind with order $r(\in \mathbb{N})$ are defined by the generating function to be 


$$
\begin{aligned}
& \int_{\mathbb{Z}_{p}} \cdots \int_{\mathbb{Z}_{p}}(1+t)^{-x_{1}-\cdots-x_{r}-x} d \mu_{-1}\left(x_{1}\right) \cdots d \mu_{-1}\left(x_{r}\right) \\
& =\left(\frac{2}{2+t}\right)^{r}(1+t)^{r}(1+t)^{x}=\sum_{n=0}^{\infty} \hat{C h}_{n}^{(r)}(x) \frac{t^{n}}{n !} .
\end{aligned}
$$

In special case, when $x=0, \hat{C h}{ }_{n}^{(r)}=\hat{C h} h_{n}^{(r)}(0)$ are called the Changhee numbers of the second kind with order $r$.

Let $f(t)$ be a formal power series in the indeterminate $t$, then $f(t)$ has the form

$$
f(t)=\sum_{k=0}^{\infty} f_{k} t^{k}
$$

As usual, the coefficient of $t^{n}$ in $f(t)$ may be denoted by $\left[t^{n}\right] f(t)$.

A Riordan array is a pair $(g(t), f(t))$ of formal power series with $f_{0}=f(0)=0$. It defines an infinite lower triangular array $\left(d_{n, k}\right)_{n, k} \in \mathbb{N}$ according to the rule:

$$
d_{n, k}=\left[t^{n}\right] g(t)(f(t))^{k} \text {. [5] [6] [7] [8] [9] }
$$

Hence we write $\mathfrak{R}\left(d_{n, k}\right)=(g(t), f(t))$.

Lemma 1 If $D=(g(t), f(t))=\left(d_{n, k}\right)_{n, k \in \mathbb{N}}$ is an Riordan array and $h_{\infty}(t)$ is the generating function of the sequence $\left\{\left(h_{k}\right)_{k \in \mathbb{N}}\right\}$, i.e.,. $f(t)=\sum_{k=0}^{\infty} f_{k} t^{k}$ or $h(t)=G\left(h_{k}\right)$. Then we have

$$
\sum_{k=0}^{n} d_{n, k} h_{k}=\left[t^{n}\right] g(t) h(f(t)) .[7]
$$

For convenience, we recall some definitions in the paper. The generalized Stirling numbers of the second kind $S(n, k ; r)$ have the following exponential generating function [6]:

$$
\sum_{n \geq k} S(n, k ; r) \frac{t^{n}}{n !}=e^{r t} \frac{\left(e^{t}-1\right)^{k}}{k !} .
$$

The higher-order Changhee polynomials $C h_{n}^{(r)}(x)$ may be related to the generalized Genocchi polynomials $G_{n}^{(\alpha)}(x)$ and the generalized Genocchi numbers $G_{n}^{(\alpha)}$, which are defined by the generating function [7] to be:

$$
\begin{aligned}
& \sum_{n \geq 0} G_{n}^{(\alpha)}(x) \frac{t^{n}}{n !}=\left(\frac{2 t}{e^{t}+1}\right)^{\alpha} e^{x t}, \\
& \sum_{n \geq 0} G_{n}^{(\alpha)} \frac{t^{n}}{n !}=\left(\frac{2 t}{e^{t}+1}\right)^{\alpha} .
\end{aligned}
$$

Another two interesting numbers, associated with the higher-order Daehee numbers of both kinds are defined by the generating function [8] to be:

$$
\begin{gathered}
\sum_{n \geq 0} D_{n}^{(r)} \frac{t^{n}}{n !}=\left(\frac{\ln (1+t)}{t}\right)^{r}, \\
\sum_{n \geq 0} \hat{D}_{n}^{(r)} \frac{t^{n}}{n !}=\left(\frac{(1+t) \ln (1+t)}{t}\right)^{r} .
\end{gathered}
$$

where $D_{n}^{(r)}$ are the higher-order Daehee numbers of the first kind and $\hat{D}_{n}^{(r)}$ 
are the higher-order Daehee numbers of the second kind.

The generating functions of generalized harmonic polynomials $H_{n}^{(r)}(x)$ are given by [5]:

$$
\sum_{n \geq 0} H_{n}^{(r)}(z) t^{n}=\frac{(-\ln (1-t))^{r+1}}{t(1-t)^{1-z}} .
$$

The generating functions of higher-order Euler polynomials $E_{n}^{(r)}(x)$ are defined by [3]:

$$
\sum_{n \geq 0} E_{n}^{(r)} \frac{t^{n}}{n !}=\left(\frac{2}{e^{z}+1}\right)^{r} e^{x t}
$$

\section{Identities of Changhee Numbers and Special Combinatorial Sequences}

Theorem 2.1 For $n \geq 0$, the following relations hold:

$$
\begin{aligned}
& \sum_{k=0}^{n} L(n, k ; i) C h_{k}^{(r)}=\hat{C} h_{n}^{(r)}(i), \\
& \sum_{k=0}^{n} L(n, k ; i) \hat{C h_{k}^{(r)}}=C h_{n}^{(r)}(i) .
\end{aligned}
$$

Proof An interesting Riordan arrays, associated with the Lah numbers $L(n, k ; r)$ are defined by

$$
\Re\left(\frac{k !}{n !} L(n, k ; r)\right)=\left((1+t)^{r}, \frac{-t}{1+t}\right) .
$$

Then applying the summation property (11) to the Riordan array (21) and the generating function (5) yields

$$
\begin{aligned}
\sum_{k=0}^{n} \frac{k !}{n !} L(n, k ; r) \frac{1}{k !} C h_{k}^{(r)} & =\left[t^{n}\right](1+t)^{i}\left[\left(\frac{2}{2+y}\right)^{r} \mid y=\frac{-t}{1+t}\right] \\
& =\left[t^{n}\right](1+t)^{i}\left\{\frac{2}{2+t}(1+t)\right\}^{r}=\hat{C h}_{n}^{(r)}(i) .
\end{aligned}
$$

from which we can establish Equation (19).

Similarly, from the Riordan array (21) and the generating function (8), we can get the Equation (20). Then the proof is complete.

For $r, k \in \mathbb{N}$, when $n>r+k$, the combinatorial numbers $P(r, n, k)$ defined by the following generating functions:

$$
\sum_{n=k}^{\infty}\left(\begin{array}{l}
n \\
k
\end{array}\right) P(r, n, k) t^{n-k}=\frac{[-\ln (1-t)]^{r}}{(1-t)^{k+1}},
$$

then Equation (22) is equivalent to

$$
\sum_{n=0}^{\infty}\left(\begin{array}{c}
n+k \\
k
\end{array}\right) P(r, n+k, k) t^{n}=\frac{[-\ln (1-t)]^{r}}{(1-t)^{k+1}},
$$

Based on the generating function (22), we obtain the next Riordan arrays, to which we pay particular attention in the next Theorem: 


$$
\mathfrak{R}\left\{\left(\begin{array}{l}
n \\
k
\end{array}\right) P(r, n, k)\right\}=\left(\frac{[-\ln (1-t)]^{r}}{1-t}, \frac{t}{1-t}\right) .
$$

Theorem 2.2 For $n>r \geq 0$, we have

$$
\sum_{k=0}^{n}\left(\begin{array}{l}
n \\
k
\end{array}\right) \frac{2^{k}}{k !} C h_{k}^{(r+1)} P(r, n, k)=(-1)^{n-r} \frac{\hat{D}_{n-r}^{(r)}}{(n-r) !} .
$$

Proof Applying the summation property (11) to the Riordan array (24) and the generating function (5) yields

$$
\begin{aligned}
& \sum_{k=0}^{n}\left(\begin{array}{l}
n \\
k
\end{array}\right) \frac{2^{k}}{k !} C h_{k}^{(r+1)} P(r, n, k) \\
& =\left[t^{n}\right] \frac{[-\ln (1-t)]^{r}}{1-t}\left[\left(\frac{1}{1+y}\right)^{r+1} \mid y=\frac{t}{1-t}\right] \\
& =\left[t^{n}\right] \frac{[\ln (1-t)]^{r}(1-t)^{r}}{(-t)^{r}} \cdot t^{r}=(-1)^{n-r} \frac{\hat{D}_{n-r}^{(r)}}{(n-r) !},
\end{aligned}
$$

Which gives Equation (25).

Theorem 2.3 For $n, i, s, r \in \mathbb{N}$, we have

$$
\sum_{k=0}^{n} \frac{(-2)^{n-k}}{(n-k) !}\left(\begin{array}{c}
k+s \\
s
\end{array}\right) C h_{n-k}^{(r)} P(i, k+s, s)=\left(\begin{array}{c}
n+s+r \\
s+r
\end{array}\right) P(i, n+s+r, s+r) .
$$

Proof Based on the generating functions (5), (6) and (7), we obtain the next Riordan arrays:

$$
\mathfrak{R}\left((-2)^{n-k} \frac{C h_{n-k}^{(r)}}{(n-k) !}\right)=\left(\frac{1}{(1-t)^{r}}, t\right) .
$$

To obtain Equation (26), apply the summation property (11) to the Riordan array (27) and the generating functions (23), we have

$$
\begin{aligned}
& \sum_{k=0}^{n} \frac{(-2)^{n-k}}{(n-k) !}\left(\begin{array}{c}
k+s \\
s
\end{array}\right) C h_{n-k}^{(r)} P(i, k+s, s) \\
& =\left[t^{n}\right] \frac{1}{(1-t)^{r}}\left[\frac{[-\ln (1-y)]^{i}}{(1-y)^{s+1}} \mid y=t\right] \\
& =\left(\begin{array}{c}
n+s+r \\
s+r
\end{array}\right) P(i, n+s+r, s+r),
\end{aligned}
$$

which gives Equation (26).

Corollary 2.1 For $n, r, i \in \mathbb{N}$, the following relations hold:

$$
\sum_{k=0}^{n}(-2)^{n-k}\left(\begin{array}{l}
n \\
k
\end{array}\right)|s(k+1, i+1)| C h_{n-k}^{(r)}=\frac{n !}{i !}\left(\begin{array}{c}
n+r \\
r
\end{array}\right) P(i, n+r, r) .
$$

Proof From the generating functions of unsigned Stirling numbers of the first kind

$$
\sum_{n \geq k}|s(n, k)| \frac{t^{n}}{n !}=\frac{[-\ln (1-t)]^{k}}{k !},
$$

we obtain the next Riordan arrays 


$$
\mathfrak{R}\left(\frac{k !}{n !}|s(n, k)|\right)=(1,-\ln (1-t)),
$$

From Equation (29), it can be verified that

$$
\sum_{n=0}^{\infty} \frac{k !}{n !} s(n+1, k+1) \mid t^{n}=\frac{[-\ln (1-t)]^{k}}{1-t} .
$$

Thus, comparing with Equation (22), we obtain the following connection between the numbers $P(r, n, k)$ and the unsigned Stirling numbers of the first kind: $P(k, n, 0)=\frac{k !}{n !}|s(n+1, k+1)|$, Finally, the substitution $s=0$ in Equation (26) gives Equation (28).

Corollary 2.2 For $n, r, s \in \mathbb{N}$, we have

$$
\begin{gathered}
\sum_{k=0}^{n} \frac{(-2)^{n-k}}{(n-k) !}\left(\begin{array}{c}
k+s \\
s
\end{array}\right) C h_{n-k}^{(r)}=\left(\begin{array}{c}
n+r+s \\
r+s
\end{array}\right), \\
\sum_{k=0}^{n} \frac{(-2)^{n-k}}{(n-k) !}\left(\begin{array}{c}
k+s \\
s
\end{array}\right) C h_{n-k}^{(r)}\left(H_{k+s}-H_{s}\right)=\left(\begin{array}{c}
n+r+s \\
r+s
\end{array}\right)\left(H_{n+r+s}-H_{r+s}\right), \\
\sum_{k=0}^{n} \frac{(-2)^{n-k}}{(n-k) !}\left(\begin{array}{c}
k+s \\
s
\end{array}\right) C h_{n-k}^{(r)}\left[\left(H_{k+s}-H_{s}\right)^{2}-\left(H_{k+s}^{(2)}-H_{s}^{(2)}\right)\right] \\
=\left(\begin{array}{c}
n+r+s \\
r+s
\end{array}\right)\left[\left(H_{n+r+s}-H_{r+s}\right)^{2}-\left(H_{n+r+s}^{(2)}-H_{r+s}^{(2)}\right)\right] .
\end{gathered}
$$

Proof Setting $i=0, i=1, i=2$ in Theorem 2.3 gives Equation (30), (31), (32), respectively.

Let $P_{n, k}(z)$ are the generalized Stirling polynomials of the first kind defined by [2]:

$$
P_{n, k}(z)=\sum_{j=k+1}^{n}(-z)^{j-k-1}\left(\begin{array}{c}
j-1 \\
k
\end{array}\right)|s(n, j)|,
$$

with the alternative representation:

$$
P_{n, k}(z)=\frac{(-1)^{k}}{k !}\left(\frac{\partial^{n-1}}{\partial t^{n-1}} \frac{\ln ^{k}(1-t)}{(1-t)^{1-z}} \mid t=0\right) .
$$

Now, let us define the infinite lower triangular martrice $P(z)$ by:

$$
P(z)=\left\{\frac{k !}{n !} P_{n+1, k}(z)\right\}_{n, k \in N_{0}},
$$

It is easy to show that $P(z)$ may be expressed by the Riordan array:

$$
\Re\left(\frac{k !}{n !} P_{n+1, k}(z)\right)=\left(\frac{1}{(1-t)^{1-z}},-\ln (1-t)\right) .
$$

Theorem 2.4 For $n \geq 0$, the following relations hold:

$$
\begin{aligned}
\sum_{k=0}^{n} P_{n+1, k}(z) G_{k}^{(i+1)} & =n ! \sum_{j=0}^{n-1} \frac{(-1)^{j}}{j !} \hat{C h}{ }_{j}^{(i+1)} H_{n-j-1}^{(i)}(z) \\
& =n ! \sum_{j=0}^{n-1} \frac{(-1)^{j}}{j !} \hat{C h}{ }_{j}^{(i+1)}(z) H_{n-j-1}^{(i)}(2 z) .
\end{aligned}
$$


Proof To obtain Equation (34), from the Riordan array (33) and the generating function (14), (17), we have

$$
\begin{aligned}
& \sum_{k=0}^{n} \frac{k !}{n !} P_{n+1, k}(z) \frac{1}{k !} G_{k}^{(i+1)}=\left[t^{n}\right] \frac{1}{(1-t)^{1-z}}\left[\left(\frac{2 y}{e^{y}+1}\right)^{i+1} \mid y=-\ln (1-t)\right] \\
& =\left[t^{n-1}\right] \frac{[-\ln (1-t)]^{i+1}}{t(1-t)^{1-z}}\left(\frac{2}{2-t}\right)^{i+1}(1-t)^{i+1}=\sum_{j=0}^{n-1} \frac{(-1)^{j}}{j !} \hat{C h}_{j}^{(i+1)} H_{n-j-1}^{(i)}(z) .
\end{aligned}
$$

Similarly, from the Riordan array (33) and the generating function (14), then we can get the Equation (34),

$$
\begin{aligned}
& \sum_{k=0}^{n} \frac{k !}{n !} P_{n+1, k}(z) \frac{1}{k !} G_{k}^{(i+1)}=\left[t^{n}\right] \frac{1}{(1-t)^{1-z}}\left[\left(\frac{2 y}{e^{y}+1}\right)^{i+1} \mid y=-\ln (1-t)\right] \\
& \pm=\sum_{j=0}^{n-1} \frac{(-1)^{j}}{j !} \hat{C h}_{j}^{(i+1)}(z) H_{n-j-1}^{(i)}(2 z) .
\end{aligned}
$$

Which completes the proof.

\section{Identities Involving the Changhee Polynomials}

Theorem 3.1 For $n \geq 0$, we have

$$
C h_{n}^{(k)}(x)=\sum_{i=0}^{n}\left(\begin{array}{l}
n \\
i
\end{array}\right)(x)_{n-i} C h_{i}^{(k)} .
$$

Proof From generating functions (5), we have

$$
\sum_{n=0}^{\infty} C h_{n}^{(k)}(x) \frac{t^{n}}{n !}=\left(\frac{2}{2+t}\right)^{k}(1+t)^{x}=\sum_{n=0}^{\infty} \sum_{i=0}^{n}\left(\begin{array}{l}
n \\
i
\end{array}\right)(x)_{n-i} C h_{i}^{(k)} \frac{t^{n}}{n !} .
$$

Comparing the coefficients of $\frac{t^{n}}{n !}$ on both sides, we obtain Equation (35). Which completes the proof.

Theorem 3.2 For $n \geq 0$, we have

$$
C h_{n}^{(r+s)}(x+y)=\sum_{i=0}^{n}\left(\begin{array}{l}
n \\
i
\end{array}\right) C h_{i}^{(r)}(x) C h_{n-i}^{(s)}(y) .
$$

Proof From the generating functions (5), we have

$$
\begin{aligned}
\sum_{n=0}^{\infty} C h_{n}^{(r+s)}(x+y) \frac{t^{n}}{n !} & =\left(\frac{2}{2+t}\right)^{r}(1+t)^{x}\left(\frac{2}{2+t}\right)^{s}(1+t)^{y} \\
& =\sum_{n=0}^{\infty} \sum_{i=0}^{n}\left(\begin{array}{c}
n \\
i
\end{array}\right) C h_{i}^{(r)}(x) C h_{n-i}^{(s)}(y) \frac{t^{n}}{n !} .
\end{aligned}
$$

Comparing the coefficients of $\frac{t^{n}}{n !}$ on both sides, we obtain Equation (36).

Which completes the proof.

Corollary 3.1 The following relations hold:

$$
C h_{n}^{(r)}(x+y)=\sum_{i=0}^{n}\left(\begin{array}{l}
n \\
i
\end{array}\right) C h_{i}^{(r)}(x)(y)_{n-i} .
$$

Proof Setting $s=0$ in Theorem 3.2, we can get Equation (37). 
The generalized harmonic numbers $H_{n, k}$ have the following exponential generating function:

$$
\sum_{n=0}^{\infty} H_{n, k} t^{n}=\frac{[-\ln (1-t)]^{r}}{r !(1-r)}
$$

Let us define $\mathfrak{h}=\left[H_{n, r}\right]_{n \geq r \geq 0}$ to be an infinite lower triangular array, it is easy to show that $\mathfrak{h}$ does not constitute a Riordan array but

$$
\hat{\mathfrak{h}}=\left(r !(-1)^{n+r} H_{n, r}\right)=\left(\frac{1}{1+t}, \ln (1+t)\right),
$$

is a Riordan array.

Theorem 3.3 Let $n \geq 0$, then

$$
\sum_{k=0}^{n}(-1)^{n+k} H_{n, k} E_{k}^{(r)}(x)=\frac{C h_{n}^{(r)}(x-1)}{n !} .
$$

Proof From the Riordan array (38) and the generating function (18), we have

$$
\begin{aligned}
\sum_{k=0}^{n} k !(-1)^{n+k} H_{n, k} \frac{E_{k}^{(r)}(x)}{k !} & =\left[t^{n}\right] \frac{1}{1+t}\left[\left(\frac{2}{1+e^{y}}\right)^{r} e^{x y} \mid y=\ln (1+t)\right] \\
& =\left[t^{n}\right]\left(\frac{2}{2+t}\right)^{r}(1+t)^{x-1} \\
& =\frac{C h_{n}^{(r)}(x-1)}{n !} .
\end{aligned}
$$

Which completes the proof.

Corollary 3.2 When $r=1$, we have

$$
\sum_{k=0}^{n}(-1)^{n+k} H_{n, k} E_{k}(x)=\frac{C h_{n}^{(r)}(x-1)}{n !} .
$$

Theorem 3.4 For $n \geq 0$, the higher-order Euler polynomials $E_{n}^{(r)}(x)$ may be expressed by means of the Changhee polynomials of both kinds,

$$
\begin{gathered}
E_{n}^{(r)}(x+r)=\sum_{k=0}^{n} C h_{k}^{(r)}(x) S(n, k ; r), \\
E_{n}^{(r)}(x+2 r)=\sum_{k=0}^{n} \hat{C} h_{k}^{(r)} S(n, k ; r) .
\end{gathered}
$$

Proof From Equation (12), we note that the generalized Stirling numbers of second kind $(S(n, k ; r))_{n, k \in \mathbb{N}}$ may be expressed by the Riordan array:

$$
\mathfrak{R}\left(\frac{k !}{n !} S(n, k ; r)\right)=\left(e^{r t}, e^{t}-1\right) .
$$

To obtain Equation (40), from the Riordan array (42) and the generating function (5), we have

$$
\begin{aligned}
\sum_{k=0}^{n} \frac{C h_{k}^{(r)}(x)}{k !} \frac{k !}{n !} S(n, k ; r) & =\left[t^{n}\right] e^{r t}\left(\frac{2}{2+y}\right)^{r}\left[(1+y)^{x} \mid y=e^{t}-1\right] \\
& =\left[t^{n}\right]\left(\frac{2}{1+e^{t}}\right)^{r} e^{t(x+r)}=\frac{E_{n}^{(r)}(x+r)}{n !} .
\end{aligned}
$$

Similarly, from the Riordan array (42) and the generating function (18), we 
can get the Equation (41).Then the proof is complete.

For a sequence $f_{n}$ and $g_{n}$, Using the inverse relation

$$
\begin{aligned}
f_{n}=\sum_{k=0}^{n} S(n, k ; r) g_{k} \Leftrightarrow & g_{n}=\sum_{k=0}^{n} s(n, k ; r) f_{k}, \text { we get } \\
& \sum_{k=0}^{n} E_{k}^{(r)}(x+r) s(n, k ; r)=C h_{n}^{(r)}(x), \\
& \sum_{k=0}^{n} E_{k}^{(r)}(x+2 r) s(n, k ; r)=\hat{C h_{n}^{(r)}(x) .}
\end{aligned}
$$

Theorem 2.5 Let $n \geq \alpha \geq 0$, we have

$$
\sum_{k=0}^{n} \sum_{j=0}^{k-\alpha}\left(\begin{array}{l}
k \\
\alpha
\end{array}\right)\left(\begin{array}{c}
k-\alpha \\
j
\end{array}\right) C h_{j}^{(\alpha)}(x-1) D_{k-j-\alpha}^{(\alpha)} S(n, k, r)=\left(\begin{array}{l}
n \\
\alpha
\end{array}\right) E_{n-\alpha}^{(\alpha)}(x+r-1) .
$$

Proof Firstly, from Equation (11), (38) and (13), we have

$$
\begin{aligned}
\sum_{k=0}^{n}(-1)^{k+n} k ! H_{n, k} \frac{G_{k}^{(\alpha)}(x)}{k !} & =\left[t^{n}\right] \frac{1}{1+t}\left[\left(\frac{2 y}{e^{y}+1}\right)^{\alpha} e^{x y} \mid y=\ln (1+t)\right] \\
& =\left[t^{n}\right]\left(\frac{2}{2+t}\right)^{\alpha}(1+t)^{x-1} \frac{(\ln (1+t))^{\alpha}}{t^{\alpha}} \cdot t^{\alpha} \\
& =\sum_{k=0}^{n-\alpha} \frac{\left(\begin{array}{c}
n-\alpha \\
k
\end{array}\right)}{(n-\alpha) !} C h_{k}^{(\alpha)}(x-1) D_{n-k-\alpha}^{(\alpha)} .
\end{aligned}
$$

Secondly, from Equation (15) and (42), we have

$$
\begin{aligned}
& \sum_{k=0}^{n} \sum_{j=0}^{k-\alpha} \frac{\left(\begin{array}{c}
k-\alpha \\
j
\end{array}\right)}{(k-\alpha) !} C h_{j}^{(\alpha)}(x-1) D_{k-j-\alpha}^{(\alpha)} \frac{k !}{n !} S(n, k ; r) \\
& =\sum_{k=0}^{n}\left[\sum_{j=0}^{k-\alpha} \frac{\left(\begin{array}{c}
k-\alpha \\
j
\end{array}\right)}{(k-\alpha) !} C h_{j}^{(\alpha)}(x-1) D_{k-j-\alpha}^{(\alpha)}\right] \frac{k !}{n !} S(n, k ; r) \\
& =\left[t^{n}\right] e^{r t}\left[\left(\frac{2}{2+y}\right)^{\alpha}(1+y)^{x-1} \ln ^{\alpha}(1+y) \mid \mathrm{y}=e^{t}-1\right] \\
& =\left[t^{n-\alpha}\right]\left(\frac{2}{1+e^{t}}\right)^{\alpha} e^{t(x+r-1)}=\frac{E_{n-\alpha}^{(\alpha)}(x+r-1)}{(n-\alpha) !} .
\end{aligned}
$$

\section{Acknowledgements}

The research is supported by the Natural Science Foundation of China under Grant 11461050 and Natural Science Foundation of Inner Mongolia under Grant 2016MS0104.

\section{References}

[1] Kim, D.S., Kim, T. and Seo, J.J. (2013) A Note on Changhee Polynomials and Numbers. Advanced Studies in Theoretical Physics, 7, 993-1003. https://doi.org/10.12988/astp.2013.39117

[2] Kwon, H.I., Kim, T. and Seo, J.J. (2015) A Note on Degenerate Changhee Numbers and Polynomials. Proceeding of the Jangjeon Mathematical Society, 18, 295-305. 
[3] Kwon, J., Noh, H.S., Jeong, S.H., Kim, A.J., Lee, J.H. and Rim, S.-H. (2015) A Note on Weighted Changhee Polynomials and Numbers. Advanced Studies in Theoretical Physics, 9, 191-198. https://doi.org/10.12988/astp.2015.410137

[4] Kim, T. and Kim, D.S. (2016) A Note on Non-Linear Changhee Differential Equations. Russian Journal of Mathematical Physics, 23, 88-92.

https://doi.org/10.1134/S1061920816010064

[5] Wang, W.S. (2015) Sum of Involving the Harmonic Numbers and the Binomial Coefficients. American Journal of Computational Mathematics, 5, 96-105. https://doi.org/10.4236/ajcm.2015.52008

[6] Cheon, G.S. and EI-Mikkawy, M.E.A. (2008) Generalized Harmonic Numbers with Riordan Array. Journal of Number Theory, 128, 413-425.

https://doi.org/10.1016/j.jnt.2007.08.011

[7] Comtet, L. (1974) Advanced Combinatorics. D. Reidel Publishing Co., Dordrecht.

[8] Kim, D.S., Kim, T., Lee, S.-H. and Seo, J.J. (2014) Higher-Order Daehee Numbers and Polynomials. International Journal of Mathematical Analysis, 8, 273-283. https://doi.org/10.12988/ijma.2014.4118

[9] Wang, W.P. and Wang, T.M. (2008) Generalized Riordan Arrays. Discrete Math, 65, 1-35. https://doi.org/10.1016/j.disc.2007.12.037 